\title{
Characteristics of Neoatherosclerosis Within Implanted Coronary Stents in Patients with Acute Coronary Syndromes
}

\author{
Edvin Benedek ${ }^{1}$, Alexandra Stănescu ${ }^{1}$, Marius Orzan² $^{2}$, Nora Rat² ${ }^{2}$ István Kovács², \\ Zsuzsanna Suciu ${ }^{2}$ \\ ${ }^{1}$ Center of Advanced Research in Multimodality Cardiac Imaging, Cardio Med Medical Center, Tîrgu Mureș, Romania \\ ${ }^{2}$ Clinic of Cardiology, University of Medicine and Pharmacy, Tîrgu Mureș, Romania
}

\section{ABSTRACT}

Introduction: In-stent restenosis (ISR) is traditionally associated with neointimal hyperplasia. However, recent studies have suggested that an underlying progression of the atherosclerotic process called neoatherosclerosis, different from intimal proliferation, could be involved in ISR development. In this study the aim was to compare the characteristics of the neoatheromatous plaque evidenced by Multislice Angio Computed Tomography, Optical Coherence Tomography (OCT) and Virtual Histology Intravascular Ultrasound (VH-IVUS) with the characteristics of de-novo lesions in native coronary vessels of patients with ISR. Material and methods: This is a prospective single-center pilot study in which patients presenting with acute chest pain and having at least one symptomatic bare-metal stent (BMS) restenosis at six months to one year after BMS implantation, were enrolled. The characteristics of the neointimal tissue developed within the implanted stents using Acio CT, OCT and VH-IVUS were studied. Results: In total, 27 patients with 38 coronary BMS were included in the study, in whom 27 ISR lesions and 43 lesions in native coronary vessels were identified. Angio CT examination revealed that atheromatous plaques responsible for ISR tend to have a larger volume compared with native lesions located in the same coronary vessel (plaque volume $91.2 \mathrm{~mm}^{3}$ for ISR vs. $60.4 \mathrm{~mm}^{3}$ for native vessels, $\mathrm{p}<0.0001$ ). Additionally, they show more low density plaques compared to native coronary lesions located in the same coronary vessel $\left(33.9 \mathrm{~mm}^{3} \mathrm{vs} .18 .2 \mathrm{~mm}^{3}\right.$ for the volume of the plaque with density <30 HU, p <0.0001). Plaques responsible for ISR exhibit a higher lipid content than native ones ( $41.1 \%$ vs. $22.9 \%$, p = 0.05). OCT analysis indicated an irregular shaped vascular lumen in $44.4 \%$ of ISR lesions compared to $25.6 \%$ of de-novo lesions $(p=0.1$ ). Conclusions: Neoatherosclerosis within the implanted coronary stents is associated with signs of plaque vulnerability to a significantly higher extent than the atheromatous plaques in native coronary arteries in patients with ISR presenting with an acute coronary syndrome.

Keywords: neoatherosclerosis, in stent restenosis, vulnerable plaque

\section{ARTICLE HISTORY}

Received: 4 September, 2015

Accepted: 16 December, 2015

\section{CORRESPONDENCE}

Alexandra Stănescu

76, 22 decembrie $1989 \mathrm{St}$

540124 Tîrgu Mureș, Romania

Tel: +40-265-217333

Email: alexandrastanescu90@gmail.com

Edvin Benedek: 76, 22 Decembrie 1989 St, 540124 Tîrgu Mureș, Romania, Tel:+40 265217 333, Email: edvinke87@gmail.com Marius Orzan: 38 Gheorghe Marinescu St, 540139 Tîrgu Mureș, Romania, Tel: +40 265215 551, Email: orzan_marius@yahoo.com Nora Rat: 38 Gheorghe Marinescu St, 540139 Tîrgu Mureș, Romania, Tel: +40 265215 551, Email: ratnora@yahoo.com Istvan Kovacs: 38 Gheorghe Marinescu St, 540139 Tîrgu Mureș, Romania, Tel: +40 265215 551, Email: kov_istvan@yahoo.com Zsuzsanna Suciu: 38 Gheorghe Marinescu St, 540139 Tîrgu Mureș, Romania, Tel: +40 265215 551, Email: suciu_zsuzsanna@yahoo.com 


\section{INTRODUCTION}

In-stent restenosis (ISR) is traditionally associated with neointimal hyperplasia. However, recent studies have suggested that a causal development of the atherosclerotic process, different from intimal proliferation, could be involved in the development of ISR. The term "neoatherosclerosis" has been recently introduced to characterize the development of a new atheromatous process within the implanted stent, extending beyond the traditional interval of several months, associated with neointimal hyperplasia. The extent to which neoatherosclerosis is an unstable condition is not clear. However, it has been reported that approximately one-third of patients with Bare-Metal Stent (BMS) restenosis present with acute coronary syndromes. Furthermore, several studies reported typical histologic morphologies in the atheromatous samples retrieved from restenotic stents, that resembled vulnerable plaques in native coronary arteries. ${ }^{1-4}$

The morphological characteristics associated with unstable plaques are well known, including positive remodeling, large lipid pools or the presence of a thin fibrous cap. Three imaging methods, most commonly used to assess the markers associated with vulnerable plaques, are Multislice Angio CT, Intravascular Ultrasound with Virtual Histology (VH-IVUS), and Optical Coherence Tomography (ОCT).

Multislice Angio CT is a non-invasive technique which provides visualisation of the coronary arteries very similar to invasive coronary angiography, the images obtained with the two techniques being completely superposable
(Figure 1). The Multislice Angio CT technique is usually favoured for non-invasive evaluation in cases of chest pain of uncertain etiology or in cases of moderate probability of coronary artery stenosis.

Intravascular ultrasound is an invasive technique based on a miniaturized transducer advanced into the coronary lumen, providing images of the coronary wall from inside the lumen. IVUS together with virtual histology softwares is used to obtain a colour codification of the coronary plaque based on the plaque composition. Using this technology, unstable components of the plaque, such as a necrotic core, are colored in red, fibrofatty tissue in yellow, fibrous component in green and calcified plaque in white (Figure 2). At the same time, it is possible to quantify exactly the amount of unstable components within the plaque, which are directly associated with the risk of rupture and the development of an acute heart attack. However, IVUS is not always suitable for the measurement of the thickness of the fibrous cap or for thrombus visualisation. ${ }^{5}$

Optical Coherence Tomography is also an invasive method, which presently is considered as the golden standard for visualisation of the apposition of the stent to the vessel wall. At the same time, the technique is considered ideal for the measurement of fibrous cap thickness, a parameter inversely related to the risk of plaque rupture (Figure 3). A thin fibrous cap is considered as a marker of risk of an acute myocardial infarction and therefore exact measurement of its thickness is crucial in predicting plaque-associated risks. OCT is also extremely useful for assessing the characteristics and morphology of restenotic tissue, which can be classified as homogenous, het-

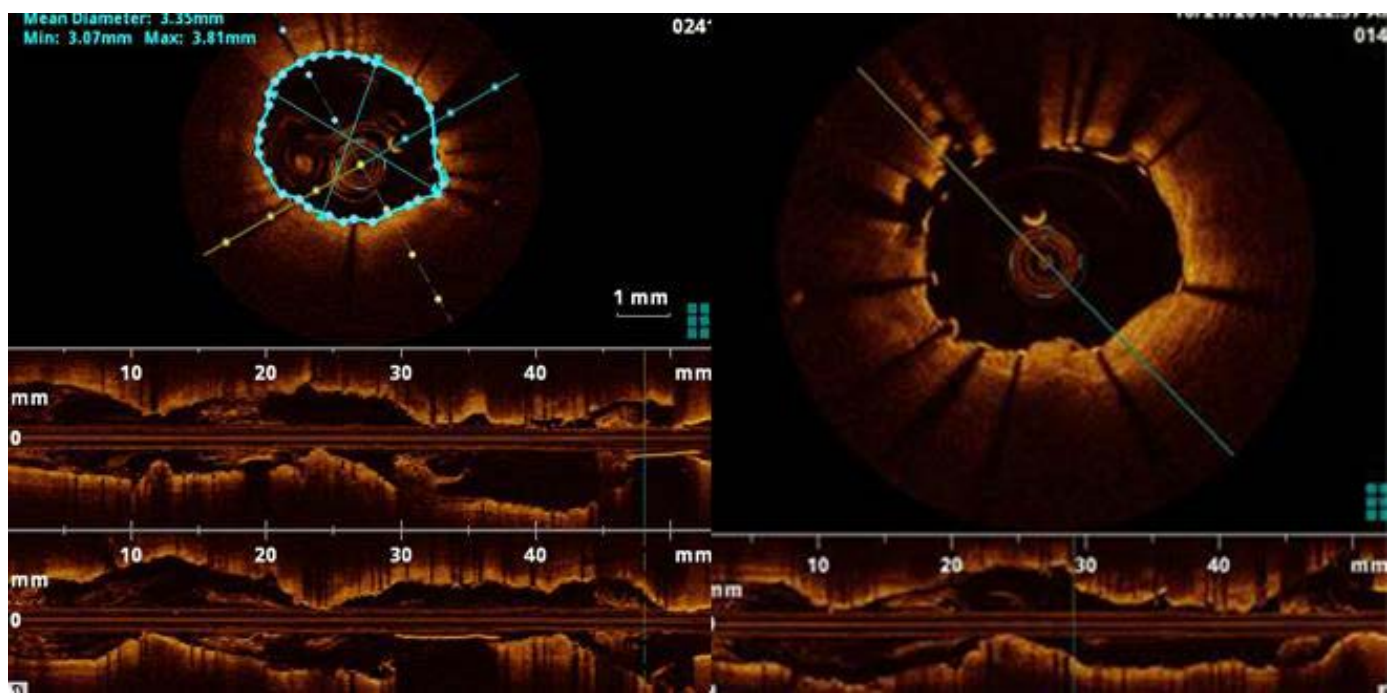

FIGURE 1. Optical Coherence Tomography: A - demonstration of the technique used for measurement of coronary lumen. B - visualisation of a coronary stent with optimal apposition to the vessel wall. 


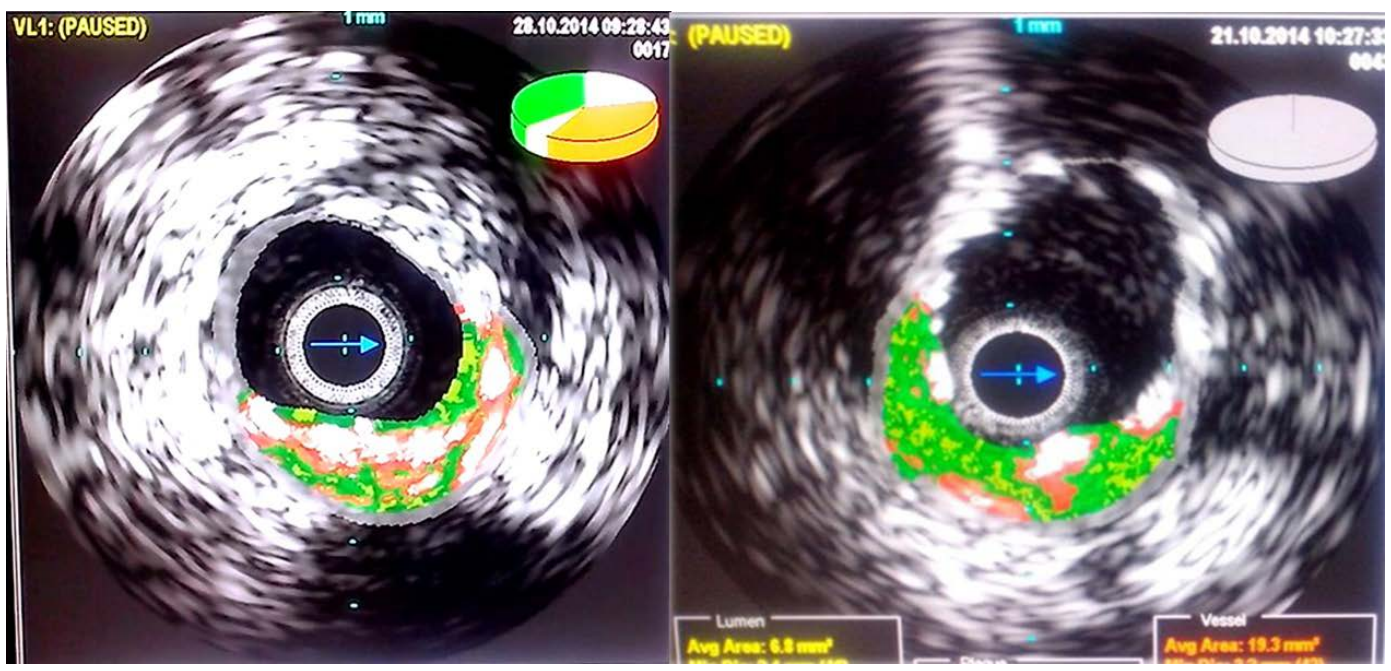

FIGURE 2. Virtual histology IVUS with colour codification of plaque composition.

erogenous or layered, with regular or irregularly shaped lumen, and with or without microvessels or without intraluminal material such as thrombi. ${ }^{6,7}$

While each of these techniques provide relevant information for plaque assessment, none of them alone provide all the required information related to atheromatous plaque vulnerability.

It is important to note that in case of a patient presenting with acute chest pain and ISR, it may be difficult to determine if the acute event is caused by an ISR lesion or by other de-novo lesions in the native coronary arteries, another lesion in the stented artery or a lesion in another coronary artery. This differentiation could be particularly important in determining a fitting therapeutic strategy and in deciding which lesion to stent in acute conditions. Combination of the information provided by the three imaging techniques can play a decisive role in the identification of an unstable plaque, i.e. the factor which produces an acute event and which should be stented in emergency conditions. ${ }^{8-11}$
In this study the aim was to compare the characteristics of neoatheromatous plaque in native coronary vessels in patients with ISR, as shown by Multislice Angio Computed Tomography, Optical Coherence Tomography (OCT) and VH-IVUS, with the characteristics of de-novo lesions.

The NULL hypothesis of this study was that there are no differences between the ISR and native vessels with respect to the composition of the atheromatous plaque as determined by OCT or VH-IVUS.

\section{MATERIAL AND METHODS}

\section{STUDY DESIGN}

This is a prospective single-center pilot study in which 27 patients with 38 coronary BMS, presenting at the emergency unit with acute chest pain and having at least one symptomatic BMS restenosis, defined as at least 50\% steno-

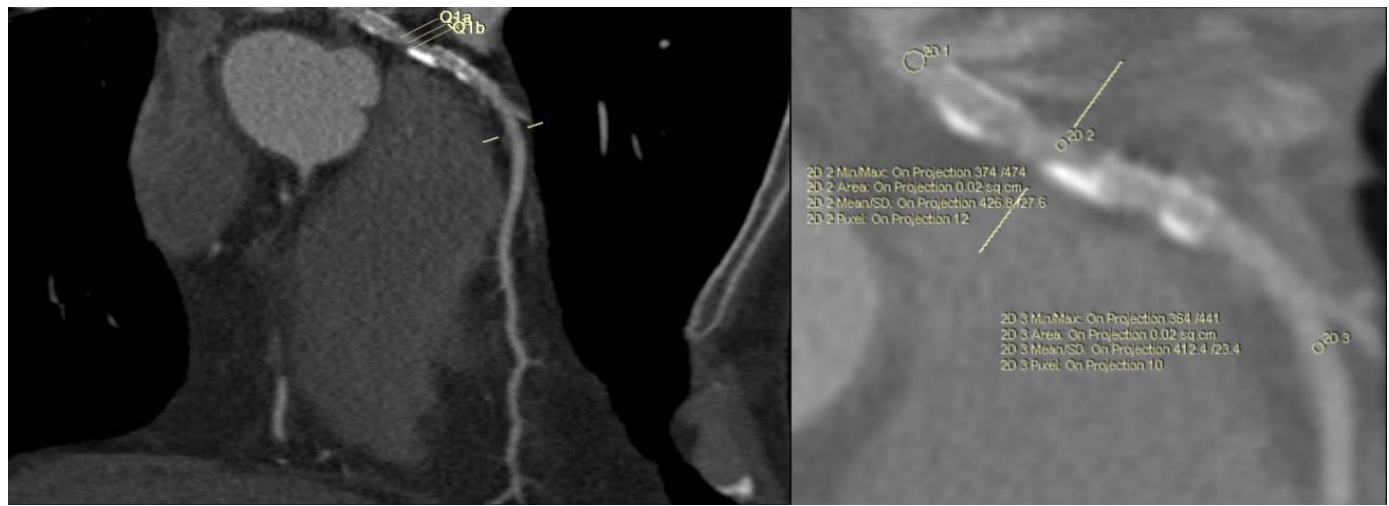

FIGURE 3. Angio CT visualisation of a coronary plaque at the level of the left anterior descending artery 
sis inside the implanted stent, identified by both Multislice Angio CT 64 and coronary angiography, six months to one year after bare metal stent implantation, were enrolled.

The study has been carried out in accordance with the code of ethics of the World Medical Association's Declaration of Helsinki. All patients gave written informed consent, and the study protocol was approved by the ethics committee of the Cardio Med Medical Center, the center where the study was conducted.

The inclusion criteria were age $>18$ years, BMS stent implanted for a de-novo coronary lesion in the last six months to one year, typical Class 3 or 4 angina as defined by the Canadian Cardiovascular Society, ECG changes suggestive of ischaemia and more than $50 \%$ diameter stenosis in the implanted stent, as shown by Multislice Angio CT and confirmed by coronary angiography. Exclusion criteria were contraindications for the repeated administration of contrast agents, previous cardiac surgery and multiple reinterventions for the same qualifying in-stent restenosis.

All patients received medication in the period following interventional revascularisation, including aspirin $75 \mathrm{mg}$, clopidogrel $150 \mathrm{mg} /$ day for seven days followed by $75 \mathrm{mg} /$ day, ACE inhibitors and $80 \mathrm{mg}$ atorvastatin in the first 30 days followed by $20 \mathrm{mg} /$ day.

All patients underwent Angio CT for assessment of vascular bed and stent patency, followed by coronary angiography with or without revascularization of the ISR lesions. Coronary angiography was used in combination with IVUS and OCT. All examinations served to assess all coronary lesions, not only those responsible for in-stent restenosis but also those in native vessels. Twenty-seven in-stent restenosis lesions and 43 lesions in native coronary vessels were identified. Plaque density was determined using Angio CT, plaque composition was assessed by IVUS associated with virtual histology, while tissue structure and fibrous cap thickness were measured by OCT.

In total 27 ISR lesions were screened and analyzed. Angio CT evaluation included visual assessment of ISR tissue shape and the presence or absence of low density areas inside the restenotic tissue. OCT assessment included measurement of the fibrous cap thickness and visual evaluation of tissue structure and shape, while IVUS assessment included quantification of the plaque components including determination of the amount of necrotic core by virtual histology.

\section{METHODOLOGY}

\section{Multislice Angio CT acquisition and interpretation}

All CT acquisitions were made using a Multi-slice 64 So- matom Sensation CT (Siemens, Erlangen, Germany) with a $64 \times 0.5 \mathrm{~mm}$ detector collimation. During an inspiratory breath-hold, $60 \mathrm{ml}$ of Iopamidol, $370 \mathrm{mg} \mathrm{I} / \mathrm{ml}$, (Bayer Healthcare, Leverkusen, Germany) was infused at a speed of $4.0 \mathrm{ml} / \mathrm{sec}$ followed by $20 \mathrm{ml}$ at $2.0 \mathrm{ml} / \mathrm{sec}$. In cases of tachycardia 50-100 $\mathrm{mg}$ of the beta-blocker metoprolol were administered. All examinations were made only after a heart rate below 70/min was attained. All acquired images were transferred to a workstation (Siemens, Erlangen, Germany) for data processing, measurements and interpretation. Plaque analysis was performed on crosssectional, longitudinal and multiplanar reformation images, and each intraluminal material was characterized by two blinded operators for the presence or the absence of an ISR, the shape of the restenotic tissue and the presence of areas inside the restenotic tissue, with a CT density less than 30 Hounsfield Units.

\section{OCT acquisition and interpretation}

OCT analysis was performed using an Iilumien ${ }^{\mathrm{TM}}$ Optis $^{\mathrm{TM}}$ PCI optimization system (St Jude medical, St Paul, Minessota, USA) immediately after coronary angiography. The following parameters were determined for each stent:

- presence of a TCFA containing neointima;

- neointimal rupture;

- thickness of the fibrous cap;

- presence of intraluminal material such as thrombi;

- restenotic tissue area;

- morphologic aspects such as homogenous, heterogenous or layered;

- shape of the lumen - regular or irregular;

- presence of a lipid pool;

- presence of microvessels;

- thrombus or macrophages.

\section{Intravascular ultrasound}

IVUS was performed using the S5 imaging system (Volcano, San Diego, USA), for the assessment of virtual histology and the quantification of the necrotic core, fibrotic tissue, fibrofatty tissue and calcifications plaque components.

Coronary angiography was performed using the Artis Zee system (Siemens Healthcare, Erlangen, UK) for diagnostic and interventional imaging.

The following parameters were determined for each stent:

- the presence of a $>50 \%$ restenosis;

- the type of restenosis, focal or diffuse. 
TABLE 1. Characteristics of the study population

\begin{tabular}{lc}
\hline & $\mathbf{n}=\mathbf{2 7}$ \\
\hline Age, years & $62.3 \pm 10.9$ \\
Gender, male & $13(48.1 \%)$ \\
Hypertension & $9(33.3 \%)$ \\
Hyperlipidemia & $18(66.6 \%)$ \\
Obesity $\left(\mathrm{BMI}>25 \mathrm{~km} / \mathrm{m}^{2}\right)$ & $8(29.6 \%)$ \\
Smoker $*$ & $11(40.7 \%)$ \\
Diabetes & $8(29.6 \%)$ \\
History of AMI & $5(18.5 \%)$ \\
History of stroke & $3(11.1 \%)$ \\
\hline
\end{tabular}

*past or present

Statistical analysis was performed using Graph InStat Pad software. Continuous values are expressed as means (SD) and statistical significance was determined using the Mann-Whitney test. Categorical variables are expressed as percentages and were compared using Fisher's exact test. The level of significance was set at $\alpha=0.05$.

\section{RESULTS}

In total, 27 patients with 38 coronary BMS were included in the study, in whom 27 ISR lesions and 43 lesions in native coronary vessels were identified. The mean age of the patients was 62.3 ( \pm 10.9 ) years and $48.1 \%$ of the patients were males. The presence of dyslipidemia was noted in $66.6 \%$ of cases, smoking status in $40.7 \%$ of cases, hypertension in $33.3 \%$ of cases, obesity in $29.6 \%$ of cases and diabetes in $29.6 \%$ of cases with ISR.

The characteristics of the patient population are presented in Table 1 . The characteristics of plaques analyzed by Angio CT, VH-IVUS and OCT are presented in Table 2.

Angio CT examination revealed that atheromatous plaques responsible for ISR tend to have a larger volume compared to native lesions located in the same coronary vessel (plaque volume $91.2 \mathrm{~mm}^{3}$ for ISR vs. $60.4 \mathrm{~mm}^{3}$ for native vessels, $\mathrm{p}<0.0001$ ) (Figure 4).

At the same time, Angio CT examination revealed that plaques responsible for ISR have a higher incidence of low density plaques, with a CT density less than 30 Hounsfield units (HU) compared to native coronary lesions located in the same coronary vessel $\left(33.9 \mathrm{~mm}^{3} \mathrm{vs}\right.$. $18.2 \mathrm{~mm}^{3}$ for the volume of plaque less than $30 \mathrm{HU}, \mathrm{p}$ $<0.0001$ ) (Figure 5).

The assessment of coronary plaques using intravascular ultrasound in conjunction with virtual histology, showed that plaques responsible for ISR have a higher lipid content than native ones ( $41.1 \%$ vs. $22.9 \%, p=0.05)$ (Figure 6 ).

OCT analysis indicated an irregularly shaped vascular lumen in $44.4 \%$ of ISR lesions compared to only $25.6 \%$

TABLE 2. Characteristics of atheromatous plaques in the ISR lesions vs. native coronary arteries

\begin{tabular}{|c|c|c|c|}
\hline & $\begin{array}{l}\text { ISR plaques } \\
\quad \mathrm{N}=27\end{array}$ & $\begin{array}{l}\text { De novo plaques } \\
\qquad N=43\end{array}$ & $\mathrm{p}$ value \\
\hline \multicolumn{4}{|l|}{ Plaque morphology } \\
\hline Irregular shape & $12(44.4 \%)$ & $11(25.6 \%)$ & 0.1 \\
\hline Heterogenous aspect & $7(25.9 \%)$ & $5(11.6 \%)$ & 0.1 \\
\hline Stenosis type - focal & $10(37.0 \%)$ & $10(23.25 \%)$ & 0.1 \\
\hline \multicolumn{4}{|l|}{ Plaque quantification } \\
\hline $\begin{array}{l}\text { Plaque volume } \\
\qquad(\mathrm{mm} 3 \pm \mathrm{SD}) \\
95 \% \mathrm{CI}\end{array}$ & $\begin{array}{c}91.2 \pm 9.28 \\
7.5-94.8\end{array}$ & $\begin{array}{c}60.4 \pm 20.35 \\
3.2-67.8\end{array}$ & $<0.0001$ \\
\hline $\begin{array}{l}\text { Low density plaque volume } \\
\qquad(\mathrm{mm} 3 \pm \mathrm{SD}) \\
\quad 95 \% \mathrm{CI}\end{array}$ & $\begin{array}{l}33.9 \pm 8.9 \\
30.9-37.5\end{array}$ & $\begin{array}{c}18.2 \pm 6.2 \\
16.3-20.16\end{array}$ & $<0.0001$ \\
\hline $\begin{array}{l}\text { Lipid burden } \\
\qquad(\% \pm \mathrm{SD}) \\
95 \% \mathrm{CI}\end{array}$ & $\begin{array}{c}41.1 \pm 9.7 \\
37.2-44.9\end{array}$ & $\begin{array}{c}22.9 \pm 10.4 \\
19.7-26.1\end{array}$ & $<0.0001$ \\
\hline Vulnerability markers & & & \\
\hline Low density plaque & $17(62.9 \%)$ & $12(27.9 \%)$ & 0.005 \\
\hline Thickness of the fibrous cap $<100 \mu \mathrm{m}$ & $13(48.1 \%)$ & $4(9.3 \%)$ & 0.0004 \\
\hline $\begin{array}{l}\text { Necrotic core } \\
\quad(\% \pm \mathrm{SD}) \\
95 \% \mathrm{CI}\end{array}$ & $\begin{array}{l}22.3 \pm 5.3 \\
20.2-24.4\end{array}$ & $\begin{array}{l}11.4 \pm 4.8 \\
9.9-12.9\end{array}$ & $<0.0001$ \\
\hline
\end{tabular}




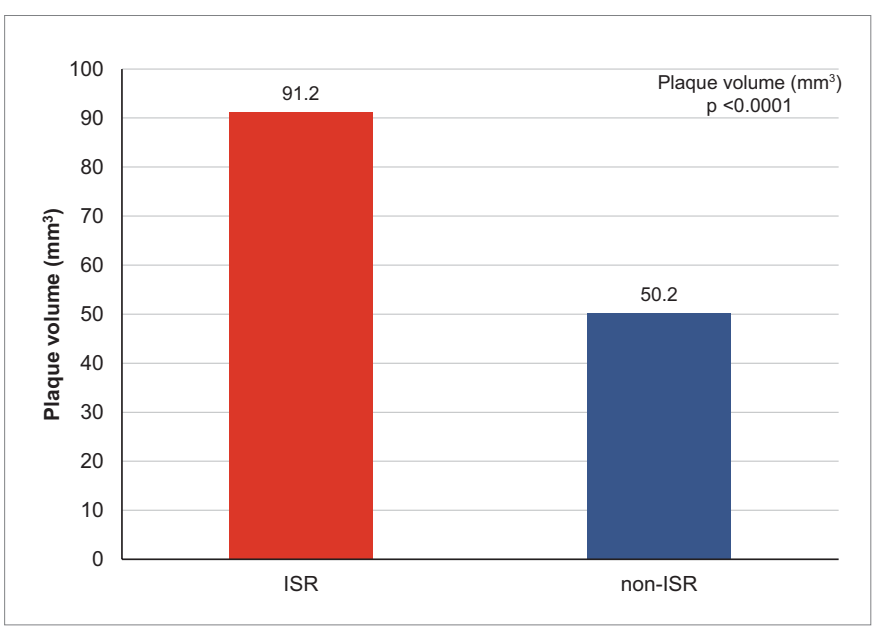

FIGURE 4. Plaque volume in neoatherosclerotic lesions vs. native lesions

in stenotic lesions in native coronary arteries $(p=0.1)$ (Figure 7).

Moreover, OCT assessment was used for the analysis of restenotic tissue patterns in plaques responsible for ISR, disclosing a heterogenous morphology in $25.9 \%$ of cases, homogenous morphology in $37 \%$ of cases and pluristratified morphology in $22.2 \%$ of cases (Figure 8 ). A fibrous cap, less than 100 um thick, as shown by OCT, was identified in 13 lesions responsible for ISR and in only 4 other lesions. Angio CT vulnerability markers such as low density plaques, with density less than $30 \mathrm{UH}$, were identified in 12 cases ( $48.1 \%$ of ISR lesions and $27.9 \%$ of native lesions, $\mathrm{p}<0.001$ ) and with VH-IVUS markers (necrotic core in $22.3 \%$ of ISR lesions and $11.4 \%$ in native coronary lesions, $\mathrm{p}=0.05$ ), while a high lipid burden of less than $40 \%$ was identified in $40.7 \%$ of ISR plaques (Figure 9).

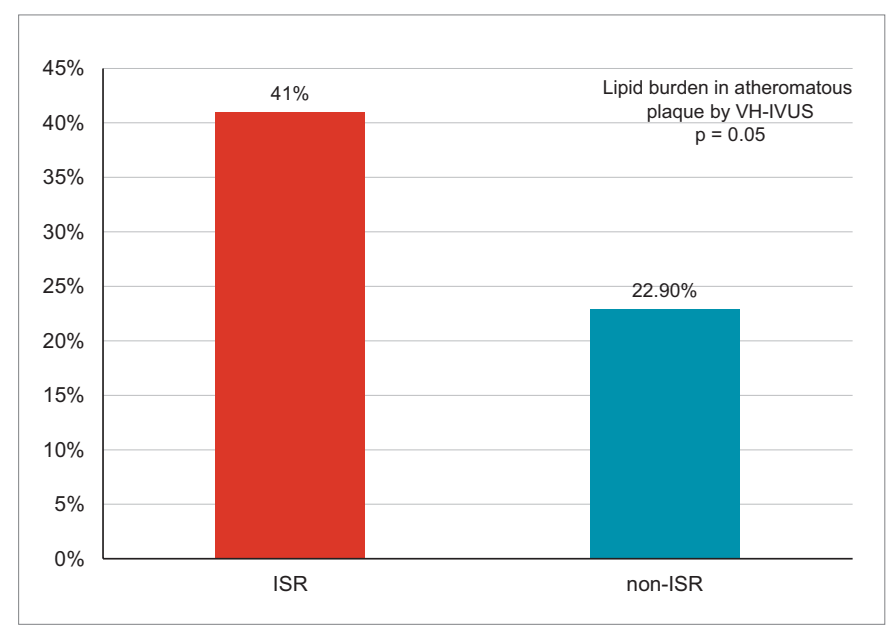

FIGURE 6. Lipid burden of atheromatous plaque in ISR lesions vs. native lesions

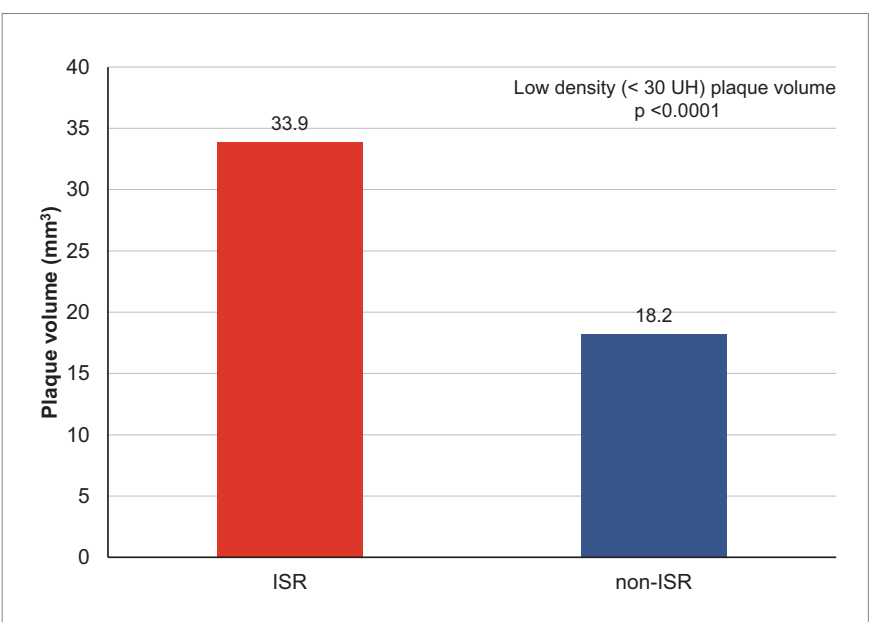

FIGURE 5. Volume of low density plaque in neoatherosclerotic lesions vs. native lesions

\section{DISCUSSION}

Atheromatous plaque progression represents a dynamic process which occurs following a stenting procedure. Neointimal hyperplasia results form various factors involving proliferation of endothelial cells consequent to balloon inflation and also to metallic stent placement, triggering alteration in laminar coronary flow. ${ }^{12,13}$

Angio CT data proved that atheromatous plaque, highly susceptible to developing restenosis, is represented by a large volume plaque with a high metabolically and endocrinologically active lipid core, and usually with a low calcification index. In general, this type of plaque could be considered chronically unstable, and the presence of areas with low density inside the plaque may indicate intensive statin therapy. ${ }^{14,15}$

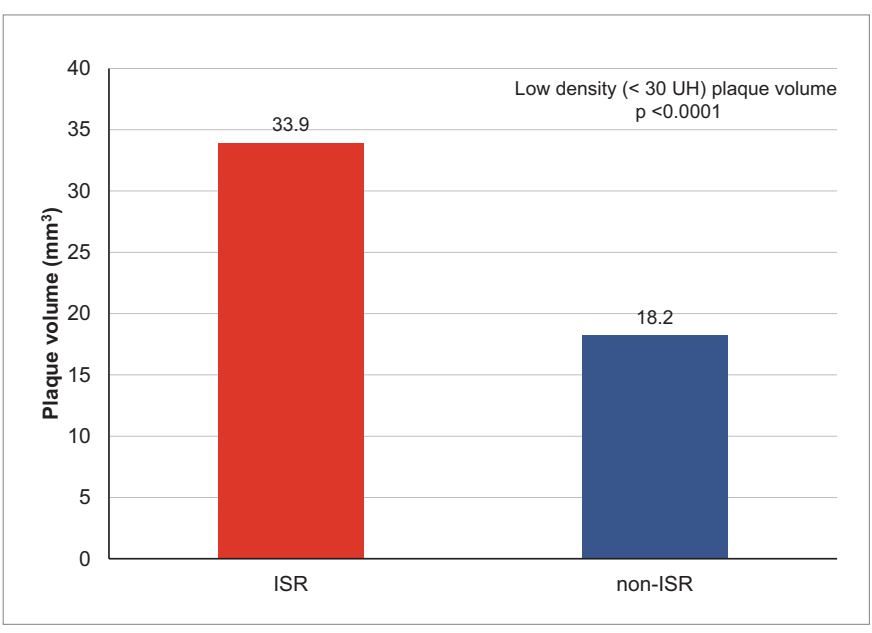

FIGURE 7. OCT-based assessment of plaque contour in ISR lesions vs. native lesions 


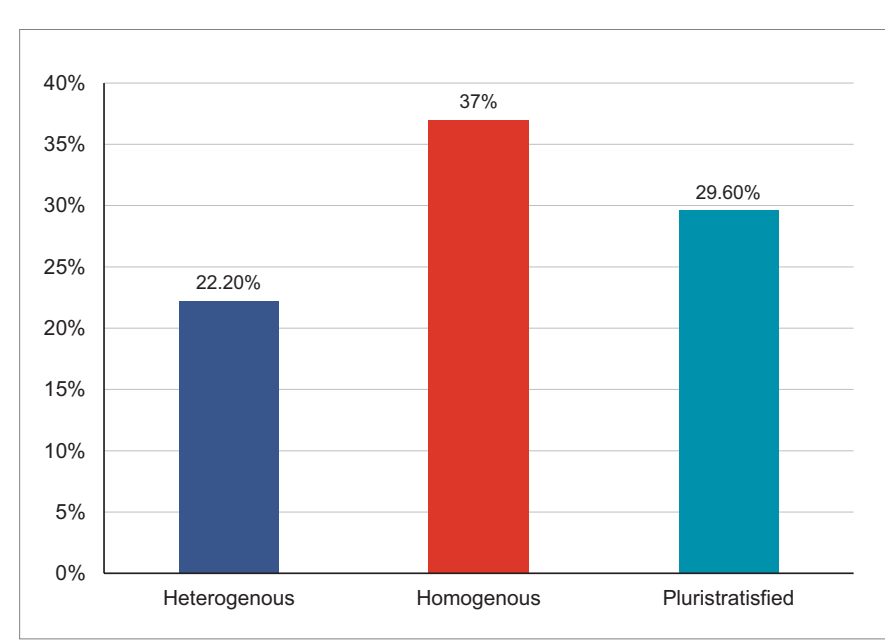

FIGURE 8. The pattern of restenostic tissue in ISR plaques

Virtual histology IVUS offers the possibility to assess the plaque composition and especially to quantify the amount of necrotic core inside the plaque, known to be highly associated with the risk of plaque rupture. At the same time, this investigation offers the possibility of providing exact quantification of vessel dimensions, plaque length and circumferential disposition of atheromatous components. This is specially important, as the ISR commences, in many cases, from the edges of the stent, associated with plaque segments not visualised by coronary angiography. ${ }^{16,17}$

OCT represents the ideal imaging method for assessing plaque vulnerability, as seen by the results of the present study, which demonstrated the presence of OCT-derived vulnerability markers to a significantly higher extent in neoatherosclerotic tissue, compared with de-novo lesions.

Modifications of intravascular geometry, caused by irregular proliferation within the implanted stents, easily determined with OCT, produces alterations in shear stress at the level of vascular wall, producing a pro-proliferative effect, probably mediated by metacrin factors. ${ }^{18}$

Motoyama (2009) showed that the presence of a low density core in coronary plaques, as identified by Angio CT, is strongly associated with the development of an acute coronary syndrome. ${ }^{19}$ Furthermore, a previous study, quantifying the extent of this vulnerable core, identified that a core of $20 \mathrm{ml}$ of very low density plaque, with less than $30 \mathrm{HU}$, was an effective indicator of the culprit lesions, in acute coronary syndromes. This very low density core is visualized by Angio CT multislice as a "dark spot" represented by dark areas with very low density within the vulnerable plaques. ${ }^{20}$

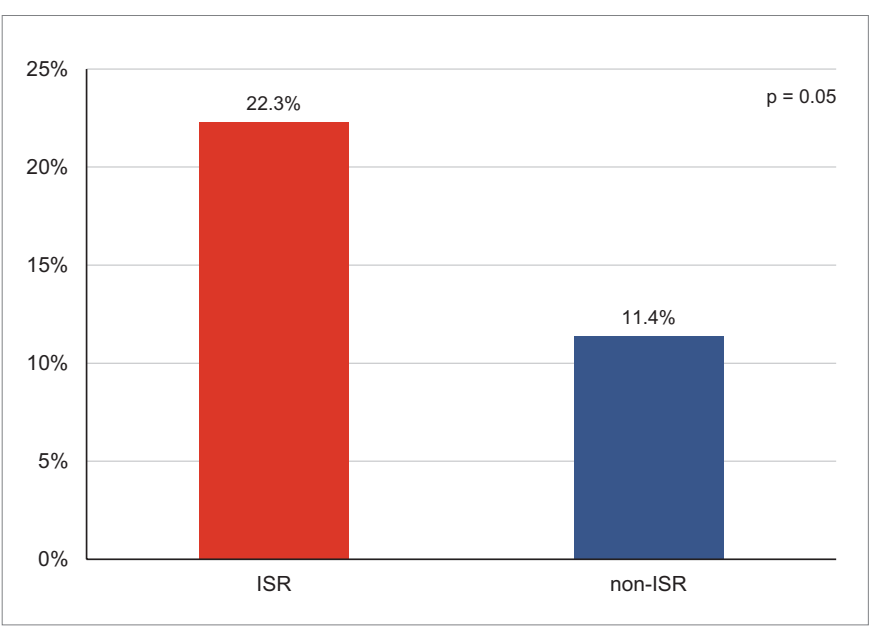

FIGURE 9. VH-IVUS determined necrotic core in ISR lesions vs. native lesions

The present study identifies the presence of vulnerability markers in the neoatherosclerotic tissue in patients with acute coronary syndomes. However the statistical significance was reached only for parameters resulting from plaque volume, volume of low density plaque, lipid burden and the percentage of necrotic core, while visual assessment was less reliable in distinguishing between vulnerable ISR tissue and de-novo lesions. This indicates that the parameters quantified by Angio CT have a higher clinical significance for predicting the vulnerability of the plaque than those based on visual assessment.

In this study it was demonstrated that neoatherosclerosis within an implanted stent represents an unstable, vulnerable plaque, and presents to a significantly higher extent, the markers associated with plaque vulnerability, compared to de-novo lesions, in native coronary arteries. The NULL hypothesis of this study, that there are no differences between the ISR and native vessels with respect to composition of atheromatous plaque as determined by OCT or VH-IVUS was rejected, as the study demonstrated clear and relevant differences between the two types of atherosclerotic plaques.

Therefore the study demonstrates that in-stent neoatherosclerosis is a different process from classical neointimal hyperplasia, in that it develops in time within the implanted coronary stents and is associated with a higher risk of acute coronary events.

\section{STUDY LIMITATIONS}

The study included a relatively small number of cases and therefore the conclusions should be generalised with caution. Further studies are required, on larger numbers, in order to validate these observations. 


\section{CONCLUSIONS}

In patients with ISR presenting with an acute coronary syndrome, neoatherosclerosis within implanted coronary stents is associated with signs of plaque vulnerability to a significantly higher extent than the atheromatous plaques in native coronary arteries. Therefore, neoatherosclerosis could be considered a process different from the classical neointimal hyperplasia, which increases the risk for acute cardiac events in patients with coronary stents. Early detection of neoatherosclerosis is possible with the help of imaging techniques such as Angio CT, IVUS and OCT, that can identify instability markers in this restenotic tissue, triggering appropriate therapeutic measures.

\section{CONFLICT OF INTEREST}

Nothing to declare.

\section{REFERENCES}

1. Chieffo A, Foglieni C, Nodari RL, et al. Histopathology of clinical coronary restenosis in drug eluting versus bare metal stents. Am J Cardiol. 2009;104:1660-1667. doi:10.1016/j. amjcard.2009.07.041.

2. Suzuki N, Nanda H, Angiolillo DJ, et al. Assessment of potential realtionship between wall shear stress and sirolimus - eluting stent implantation in patients with diabetes mellitus. Int J Cardiovasc Imaging. 2008;24:357-364. doi 10.1007/s10554007-9274-0.

3. Oikava YJ, Costa M, Matsuno S, et al. Intravasculare ultrasound, angioscopic and histopathological characterisation of heterogenous patterns of restenosis after sirolimus eluting stents implantation: insights into potential thromborestenosis phenomenon. Eurointervention. 2010;6:380-387. doi:10.1016/j. jacc.2006.04.068.

4. Gijsen FJ, Oortamn RM, WentzellJ, et al. Usefulness of shear stress pattern in predicting neointima distribution in sirolimus eluting stents in coronary arteries. Am J Cardiol. 2003;92:1325-1328. doi:10.1016/j.amjcard.2003.08.017.

5. Cook s, Ladich E, Nakazawa G, et al. Correlation of intravascular ultrasound findings with histopathological analysis of thrombus aspirates in patients with very late drug eluting stent thrombosis. Circ. 2009;120:391-399. DOI: 10.1161/CIRCULATIONAHA.109.854398.

6. Kang SJ, Mintz GS, Akasaka T, et al. Optical coherence tomographic analysis of instentneoatherosclerosis after drug eluting stent implantation. Circ. 2011;123:2954-2963. doi: 10.1161/CIRCULATIONAHA.110.988436.

7. Maisel WH. Unanswered questions - drug-eluting stents and the risk of late thrombosis. N Engl J Med. 2007;356:981-984. doi: 10.1056/NEJMp068305.

8. Van Beusekom HM, SaiaF, Zindler JD, et al. Drug-eluting stents show delayed healing: paclitaxel more pronounced than sirolimus. Eur Heart J. 2007;28:974-979. doi: http:// dx.doi.org/10.1093/eurheartj/ehmo64.

9. Kimura $\mathrm{T}$, Morimoto $\mathrm{T}$, Natsuaki $\mathrm{M}$, et al. Comparison of everolimus-eluting and sirolimus-eluting coronary stents: 1-year outcomes from the Randomized Evaluation of Sirolimus-eluting Versus Everolimus-eluting stent Trial (RESET). Circ. 2012;126:1225-1236. doi: 10.1161/ CIRCULATIONAHA.112.104059.

10. De Labriolle A, Bonello L, Lemesle G, et al. Clinical presentation and outcome of patients hospitalized for symptomatic in-stent restenosis treated by percutaneous coronary intervention: comparison between drug-eluting stents and bare metal stents. Arch Cardiovasc Dis. 2009;102:209-217. doi: 10.1016/j. acvd.2009.01.004.

11. Unverdorben M, Vallbracht C, Cremers B, et al. Paclitaxelcoated balloon catheter versus paclitaxel-coated stent for the treatment of coronaryin-stentrestenosis.Circ. 2009;119:29862994. doi: 10.1161/CIRCULATIONAHA.108.839282.

12. WentzelJJ, Gijsen FJ, Stergiopulos N, et al. Shear stress, vascular remodeling and neointimal formation. J Biomech. 2003;36:681-688. doi: http://dx.doi.org/10.1016/So0219290(02)00446-3.

13. Nakazawa G, Otsuka F, Nakano M, et al. The pathology of neoatherosclerosis in human coronary implant bare - metal and drug eluting stents. J Am Coll Cardiol. 2011;57:1314-1322. doi: 10.1016/j.jacc.2011.01.011.

14. Maurovich-Horvath P, Hoffmann U, Vorpahl M, Masataka $\mathrm{N}$, Virmani R. The napking ring: Ct signature of high risc coronary plaques. J Am Coll Cardiology Img. 2010;3:440-444. doi: 10.1016/j.jcmg.2010.02.003.

15. Rathore S, Konishita Y, Terashima M, et al. A comparation of clinical presentation, angiographic patterns and outcomes of instent restenosis between bare metal and drug eluting stents. Eurointervention. 2010;5:841-846.

16. Garcì-Garcìa HM, Gogas $\mathrm{BD}$, Serruys $\mathrm{PW}$, Bruining $\mathrm{N}$. IVUS-based imaging modalities for tissue characterization: similarities and differences. Int $\mathrm{J}$ Cardiovasc Imaging. 2011;27:215-224. doi:10.1007/s10554-010-9789-7.

17. Liu J, Maehara A, Mintz GS, et al. An integrated TAXUS IV, $\mathrm{V}$, and VI intravascular ultrasound analysis of the predictors of edge restenosis after bare metal or paclitaxel-eluting stents. Am J Cardiol. 2009;103:501-506. doi: 10.1016/j. amjcard.2008.10.010.

18. Habara M,Terashima M, Nasu K, et al. Difference of tissue characteristics between early and very late restenosis after bare metal stents implantation: an optical coherence tomography study. Circ Cardiovasc Interv. 2010;4:232-238. doi: 10.1161/CIRCINTERVENTIONS.110.959999.

19. Motoyama S, Sarai M, Harigaya H. Computed Tomographic Angiography Characteristics of Atherosclerotic Plaques Subsequently Resulting in Acute Coronary Syndrome. J Am Coll Cardiol. 2009;54:49-57. doi:10.1016/j.jacc.2009.02.068.

20. Benedek T, Gyongyosi M, Benedek I. Multislice Computed Tomographic Coronary Angiography for Quantitative Assessment of Culprit Lesions in Acute Coronary Syndromes. Canadian J Cardiol. 2013;29:364-371. doi:10.1016/j. cjca.2012.11.004. 